\title{
Automatic chatter detection in grinding
}

\author{
Janez Gradišek ${ }^{\text {a,* }}$, Andreas Baus ${ }^{\mathrm{b}}$, Edvard Govekar ${ }^{\mathrm{a}}$, Fritz Klocke ${ }^{\mathrm{b}}$, Igor Grabec ${ }^{\mathrm{a}}$ \\ a Faculty of Mechanical Engineering, University of Ljubljana, Askerceva 6, Ljubljana 1000, Slovenia \\ ${ }^{\mathrm{b}}$ Laboratory for Machine Tools and Production Engineering (WZL), RWTH Aachen, Germany
}

Received 15 November 2002; accepted 2 July 2003

\begin{abstract}
Two methods for automatic chatter detection in outer diameter plunge feed grinding are proposed. The methods employ entropy and coarse-grained information rate (CIR) as indicators of chatter. Entropy is calculated from a power spectrum, while CIR is calculated directly from fluctuations of a recorded signal. The methods are verified using signals of the normal grinding force and RMS acoustic emission. The results show that entropy and CIR perform equally well as chatter indicators. Based on the normal grinding force, they detect chatter in its early stage, while only cases of strong chatter are detected based on RMS acoustic emission.
\end{abstract} (C) 2003 Elsevier Ltd. All rights reserved.

Keywords: Grinding; Chatter; Monitoring

\section{Introduction}

Chatter vibration is an unfavorable dynamic phenomenon encountered in various machining processes. In grinding, which is often the finishing machining operation, the occurrence of chatter is particularly critical because it adversely affects the two main objectives of grinding: geometrical form accuracy and surface finish of the ground workpieces. In order to avoid additional costs due to rework or even disposal of chatter-damaged workpieces, early and reliable detection of chatter is necessary.

Regardless of its cause, chatter in grinding usually manifests itself as large amplitude, nearly harmonic vibrations of the grinding wheel and the workpiece [1]. These vibrations distort the round form of the wheel and/or the workpiece. Since wheel and workpiece macrogeometries are difficult to measure on-line in rough industrial environment, chatter detection relies mostly on the measurements of acoustic emission (AE), accelerations, or grinding forces [1].

Although recognizing the presence of chatter appears as a relatively simple task for a trained machine operator,

* Corresponding author. Tel.: +386-1-4771-166; fax: +386-12518-567.

E-mail address: janez.gradisek@fs.uni-lj.si (J. Gradišek). only a few methods of automatic chatter detection can be found in the literature (see reviews [1-3]). Most of them combine AE signals as a source of information and neural networks as a pattern recognition tool. In one of the methods [4], a back-propagation neural network was employed to detect chatter based on power spectra of the enveloped AE signal. A different approach to automatic chatter detection has been proposed recently $[5,6]$, employing a scalar indicator, the coarse-grained entropy rate (CER), calculated from the fluctuations of the normal grinding force.

This article is partly an extension of the work presented in $[5,6]$. Two chatter detection methods are proposed based on two scalar indicators: entropy, calculated from a power spectrum, and coarse-grained information rate (CIR), calculated from fluctuations of a recorded signal. The methods are verified on signals of the normal grinding force and RMS AE recorded in outer diameter plunge feed grinding. The results show that the methods perform equally well and are both appropriate for on-line automatic chatter detection. It is also shown that the normal grinding force is more sensitive to chatter vibration than $\mathrm{AE}$, providing early and more reliable detection of chatter. 


\section{Entropy and information rate}

Entropy and information rate are quantities defined in the information theory. In the following, their definitions are briefly reviewed. More details can be found in [7].

\subsection{Entropy}

Consider a discrete random variable $X$ which can attain any value from a set $\Omega=\left\{x_{1}, x_{2}, \ldots, x_{n}\right\}$, with a probability distribution denoted as $p(x)=P(X=x)$. The entropy of the variable $X$ is defined as:

$H(X)=-\sum_{x \in \Omega} p(x) \log p(x)$.

$H(x)$ quantifies the average amount of information gained from observing which $x_{i}$ from $\Omega$ actually occurred in the experiment. The values of $H(X)$ are bounded to the interval $[0, \log n]$. The upper bound is obtained for equiprobable $x_{i}$, i.e. $p\left(x_{i}\right)=1 / n$ for all $i$. On the other hand, if one particular $x_{j}$ is much more probable than all other $x_{i}, p\left(x_{j}\right) \gg p\left(x_{i}\right)$, the value of $H(X)$ is close to 0 .

For the purpose of chatter detection, power spectrum $S$ of a recorded signal is considered as a probability distribution of $X$, whereby the individual values $x_{i}$ from $\Omega$ and their probabilities $p\left(x_{i}\right)$ correspond to the frequencies $f_{i}$ and their amplitudes $s\left(f_{i}\right)$, respectively. In order to mimic the properties of a probability distribution, the amplitudes $s\left(f_{i}\right)$ have to be scaled such that their sum equals 1 . It is also convenient to normalize $H(X)$ by its maximum so that the values of $H(X)$ become bounded to the interval $[0,1]$. The normalized entropy is hereafter denoted by $H^{\prime}(X)$.

\subsection{Information rate}

Let us now consider two random variables, $X$ and $Y$, with the corresponding sets of values $\Omega_{X}$ and $\Omega_{Y}$, individual probability distributions $p(x)$ and $p(y)$, and a joint probability distribution $p(x, y)=P(X=x, Y=y)$. Analogous to Eq. (1), the joint entropy of $X$ and $Y$ is defined as:

$H(X, Y)=-\sum_{x \in \Omega_{X} \in \Omega_{Y}} p(x, y) \log p(x, y)$

The average amount of information about the variable $Y$ contained in the variable $X$ is measured by the mutual information $I(X, Y)$ :

$$
\begin{gathered}
I(X ; Y)=H(X)+H(Y)-H(X, Y) \\
=\sum_{x \in \Omega_{X}} \sum_{y \in \Omega_{Y}} p(x, y) \log \frac{p(x, y)}{p(x) p(y)} .
\end{gathered}
$$

In order to define the information rate, we turn to dynamic systems for which the variables $X$ and $Y$ are time dependent, $X(t)$ and $Y(t)$. For many experimental dynamic systems, such as a machine-tool-workpiece assembly, all the variables describing their dynamics are neither known nor measurable. However, as shown by the chaos theory $[8,9]$, it may often suffice to have access to a single variable which meaningfully reflects the system dynamics.

Now, let $X(t)$ denote the measured variable and $x(t)$ its value sampled at discrete times $t=i \Delta t, i=1,2, \ldots$. Replacing variables $X$ and $Y$ by $x(t)$ and its time delayed value $x(t+\tau)$, respectively, the norm of the mutual information can be defined as [10]:

$$
\begin{aligned}
\mathrm{CIR}= & |I(x(t) ; x(t+\tau))|= \\
& \frac{1}{\tau_{\max _{\tau}}} \sum_{\tau=\Delta \tau}^{\tau_{\max }} I(x(t) ; x(t+\tau)) \Delta \tau,
\end{aligned}
$$

with $\Delta \tau=\Delta t$ as the usual choice for the delay increment $\Delta \tau$. The maximal time delay $\tau_{\max }$ should be chosen such that $\mathrm{I}(x(t) ; x(t+\tau)) \approx 0$ for $\tau \geq \tau_{\text {max }}$.

The norm $|I(x(t) ; x(t+\tau))|$ in Eq. (4) is called the "coarse-grained information rate". The term "coarsegrained" stems from the fact that the CIR is only a coarse and relative estimate of the exact information rate which is difficult to estimate from experimental data $[10,11]$. Nevertheless, both the CIR and the exact information rate have the same physical interpretation as measures of predictability of process dynamics. The values of CIR are bounded to the interval $[0, M]$ where the upper limit $M$ is finite and depends on the way of the estimation of probability distributions (see Eq. (3)). CIR is close to 0 for random processes which "forget" their past in a single time step $\Delta t$ and are therefore not predictable at all. CIR is much higher for periodic processes which lose no information about their history and can be predicted for infinitely long time intervals.

For the results presented in this article, the probability distributions in Eq. (3) were estimated using histograms with $Q=4$ equiprobable bins for $p(x)$ and $Q^{2}=16$ bins for $p(x, y)$. The upper limit of CIR was therefore $M=$ $\log Q$. For the purpose of chatter detection, it is convenient to normalize CIR by $M$ so that its values are bounded to the interval $[0,1]$. The normalized CIR is hereafter denoted by NCIR.

CIR is related to the coarse-grained entropy rate that was used as an indicator of chatter in [5]. CER is a coarse and relative estimator of Kolmogorov-Sinai entropy of a dynamic system [8,11]. The relation between the simplest form of CER, as defined in [5], and CIR (Eq. (4)) is:

$\mathrm{CER}=\frac{I\left(x(t) ; x\left(t+\tau_{0}\right)\right)-\mathrm{CIR}}{\mathrm{CIR}}$

with $\tau_{0}=0$ as the usual choice. Since $I(x(t) ; x(t))=$ 
$H(x(t))$ is constant when probability distributions are estimated as described above, CER depends only on the CIR. It is therefore simpler and more efficient to use the CIR as a descriptor of process dynamics.

\section{Experiments}

\subsection{Setup}

Experiments with outer diameter plunge feed grinding were performed on a CNC grinding machine with conventional grinding wheels of two types (Fig. 1). Eight plunges of different depths were ground on each workpiece such that the specific material volume of $V^{\prime}{ }_{w}=$ $1040 \mathrm{~mm}^{3} / \mathrm{mm}$ was reached after the eighth plunge. Other grinding parameters were kept constant for each workpiece, but were varied between the workpieces. Specific material removal rate $Q^{\prime}{ }_{w}$ was set to $1,3,5$ and $8 \mathrm{~mm}^{3} / \mathrm{mm} \mathrm{s}$, the dressing overlap $U_{d}$ to $2,4,8$ and 16 , and the speed ratio $q$ to $-20,-50$ and -100 , where minus sign denotes the opposite directions of the workpiece and wheel revolution. Altogether 50 workpieces were ground.

Fluctuations of the normal grinding force $F_{\mathrm{n}}$ were measured using a piezoelectric force sensor mounted under the tail stock. Signals of AE were measured by a sensor mounted on a spindle of the grinding wheel. Both normal force and AE signals were initially stored on a magnetic tape and later A-D converted at sampling rates of $20 \mathrm{kHz}$ and $2 \mathrm{MHz}$, respectively, and stored on a computer for subsequent analysis. The analysis was performed on $F_{\mathrm{n}}$ signals containing 32,768 points and rootmean-square (RMS) $\mathrm{AE}$ signals, $\mathrm{AE}_{\mathrm{RMS}}$, containing 10,485 points. $\mathrm{AE}_{\mathrm{RMS}}$ signals were calculated from the raw $\mathrm{AE}$ signals using non-overlapping windows of 200 data points so that the sampling rate of $\mathrm{AE}_{\mathrm{RMS}}$ signals was $10 \mathrm{kHz}$. Both $F_{\mathrm{n}}$ and $\mathrm{AE}_{\mathrm{RMS}}$ signals were also highpass filtered with a cut-off frequency of $50 \mathrm{~Hz}$ so that the lowest harmonics of the workpiece and wheel revolution frequencies were suppressed.

Three output parameters of grinding were measured

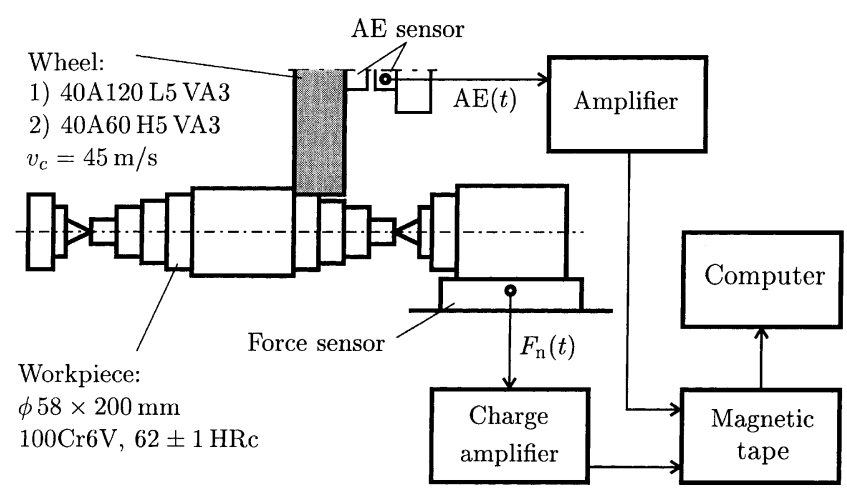

Fig. 1. Scheme of experimental setup. off-line: roundness of the workpiece, roughness of the ground surface, and radial wheel wear. Roundness and roughness were measured using a stylus-type device. The wheel was dressed prior to each workpiece.

In the following, three typical examples of chatterfree and chatter grinding regimes are presented.

\subsection{Examples of chatter-free and chatter grinding regimes}

Example 1 is from a chatter-free regime, achieved with $Q^{\prime}{ }_{w}=1 \mathrm{~mm}^{3} / \mathrm{mm} \mathrm{s}, U_{d}=4, q=-50$, and wheel 1 (see Fig. 1). The ground workpiece has a smooth profile at all the eight plunges, without any marked wavy patterns (Fig. 2). Waviness of a profile is easier to assess from its amplitude spectrum calculated by the Fourier transform. In this example, the amplitude spectra of the profiles are rather flat. Similarly, no dominant spectral peaks can be observed in amplitude spectra of the normal grinding force and RMS acoustic emission (Fig. 3), indicating only low amplitude random-like vibration during grinding.

Example 2 represents a chatter regime, achieved with $Q^{\prime}{ }_{w}=8 \mathrm{~mm}^{3} / \mathrm{mm} \mathrm{s}, U_{d}=4, q=-50$, and wheel 1 . The roundness profiles are rougher than in the chatter-free example, and they gradually become wavy as grinding progresses (Fig. 4). Pronounced waviness is observed in the last three plunges (6-8), for which chatter was also reported by the machine operator. The amplitude spectra of the profiles in plunges 1-5 show many small peaks at various integer multiples of 7 , which corresponds to the ratio of the wheel and workpiece revolution frequencies, 35.84 and $5.12 \mathrm{~Hz}$. In plunge 6, a strong peak at $n=105$ appears, and remains present also in plunges 7 and 8, together with strong peaks at $n=7$ and 14. As proved below, the wave number 105 corresponds to the ratio of the chatter vibration frequency $(537.6 \mathrm{~Hz}$ in plunge 8 ) and the workpiece revolution frequency. A strong peak dominating the spectra of the normal force is also an indication of chatter vibration (Fig. 5). In this example, the peak appears in the third plunge. In plunges 5 and 6 , its amplitude grows approximately exponentially, and remains constant in plunges 7 and 8 . The highest peak amplitudes are found in plunges with the most expressive waviness of the roundness profiles (6-8). Relatively slow growth of the peak amplitude indicates that chatter was caused by the so-called regenerative effect on the surface of the grinding wheel [1]. This assumption is also supported by the integer ratio (15) of the chatter vibration and wheel revolution frequencies, suggesting that there were 15 waves on the wheel's circumferential profile, which were, however, not measured during the experiments. A strong peak also dominates the spectra of the RMS acoustic emission signals, but only in plunges $6-8$, which correspond to the strongest, fully developed chatter vibrations. As expected, the 

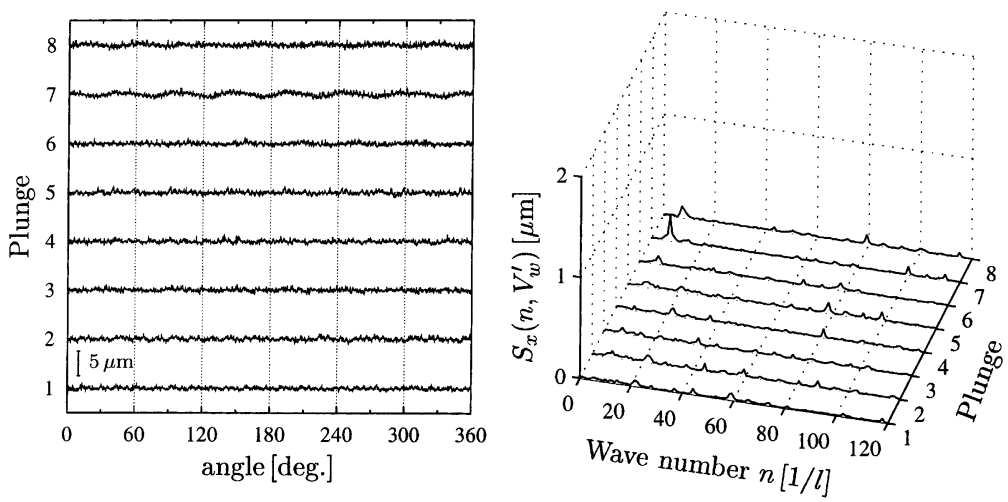

Fig. 2. Roundness profiles (left) and their amplitude spectra for the chatter-free regime (example 1). Wave number, $n$, denotes the number of waves per workpiece circumference $l$.
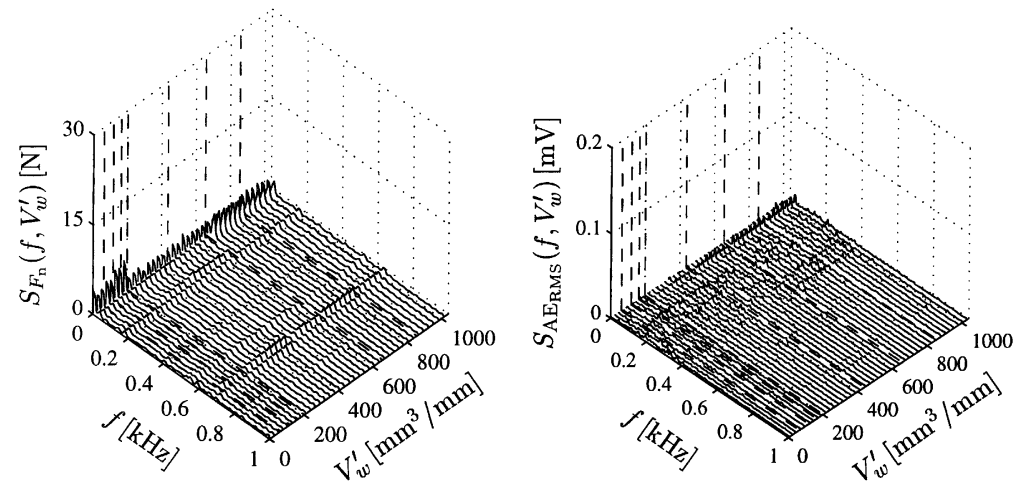

Fig. 3. Amplitude spectra of the normal force (left) and RMS acoustic emission for the chatter-free regime (example 1). Dashed lines separate the plunges.
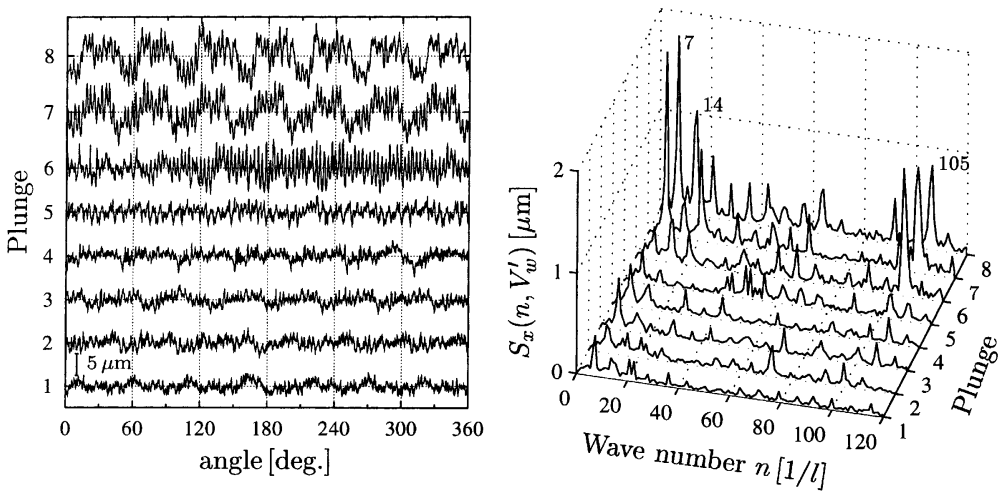

Fig. 4. Roundness profiles (left) and their amplitude spectra for the chatter regime (example 2).

peak's frequency is identical to that of the dominant peak in the normal force spectra. However, the amplitude ratio of the strongest peak and other peaks is much smaller in $\mathrm{AE}_{\mathrm{RMS}}$ than in the normal force spectra.

Example 3 is another chatter example, achieved with $Q^{\prime}{ }_{w}=3 \mathrm{~mm}^{3} / \mathrm{mm} \mathrm{s}, U_{d}=16, q=-20$, and wheel 2 . The types of chatter in examples 2 and 3 are different. The difference is revealed immediately by comparing the normal force amplitude spectra of the two examples. In example 2 (Fig. 5), the amplitude of the dominant peak grows throughout several plunges, whereas in example 3 , it grows much faster (Fig. 6). The rapid growth of the vibration amplitude is characteristic of the self-excited chatter caused by the regenerative effect on the workpiece surface [1]. Frequencies of the dominant peak, which are $534.1 \mathrm{~Hz}$ (plunge 2), $540.5 \mathrm{~Hz}$ (plunge 5), and $538.4 \mathrm{~Hz}$ (plunge 6), also provide evidence in favor of the above explanation since they are all integer multiples of the workpiece revolution frequency (43rd in plunge 2 , and 42 nd in plunges 5 and 6). Peaks at the chatter 

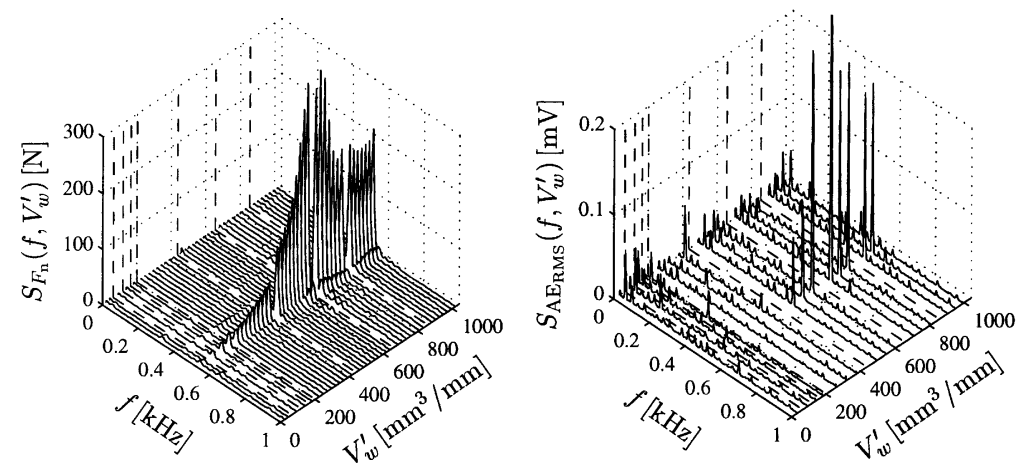

Fig. 5. Amplitude spectra of the normal force (left) and RMS acoustic emission for the chatter regime (example 2). Dashed lines separate the plunges.
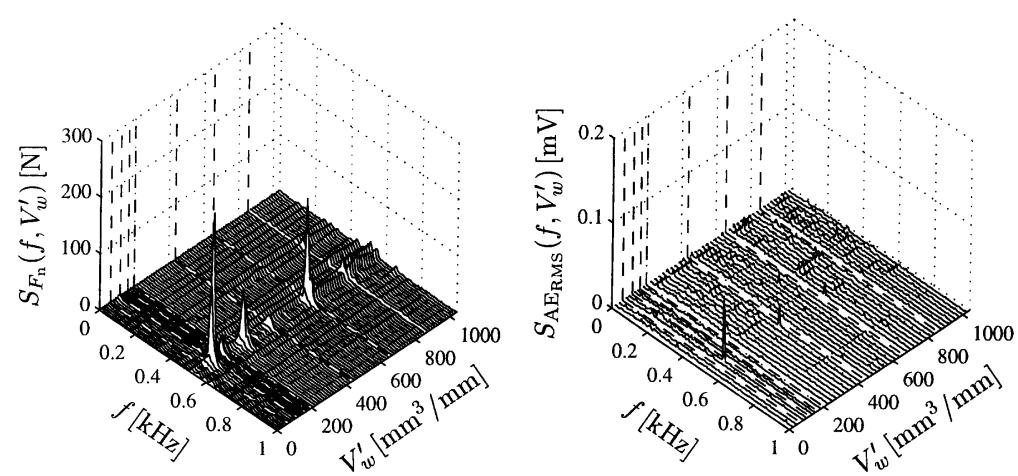

Fig. 6. Amplitude spectra of the normal force (left) and RMS acoustic emission for the chatter regime (example 3). Dashed lines separate the plunges.

frequencies are also found in the amplitude spectra of the RMS acoustic emission, but their amplitudes are small. Roundness profiles of all the eight plunges are similarly rough (Fig. 7). Slightly pronounced wavy patterns are observed in plunges 2 and 5. The amplitude spectra of the profiles show peaks at wave numbers 43 for plunge 2, 20 and 42 for plunge 5, 23 and 42 for plunge 6 , and 26 for plunge 8 . As discussed above, the numbers 43 and 42 correspond to the ratio of the chatter vibration and workpiece revolution frequencies. The wave numbers 20,23 , and 26 are close to the seventh, eighth, and ninth multiple of the ratio of wheel and workpiece revolution frequencies.

Next, the chatter detection methods proposed in Section 2 are verified on the signals of the normal grinding force and RMS acoustic emission from the three examples presented.

\section{Chatter detection results}

First, the normalized entropy of a power spectra $H^{\prime}(S)$ is considered as an indicator of chatter. As shown in the
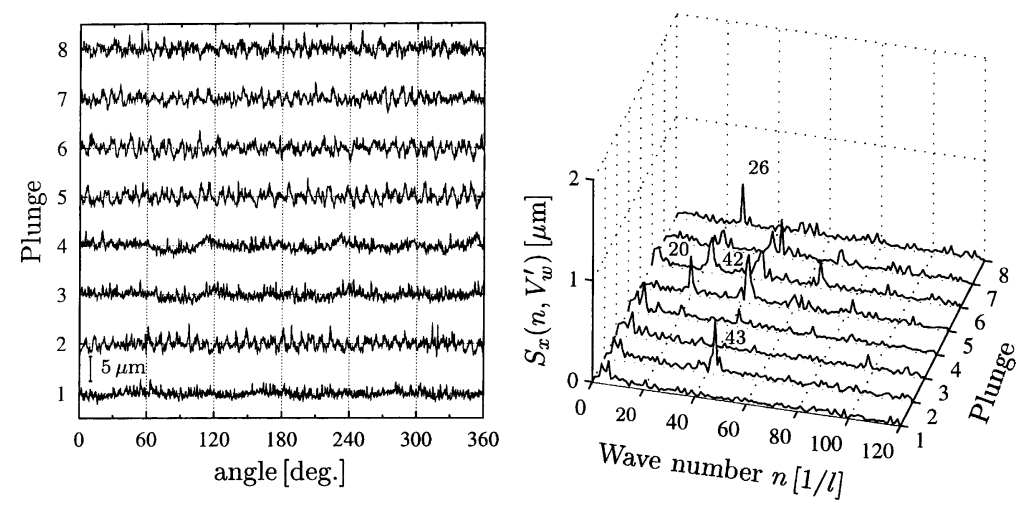

Fig. 7. Roundness profiles (left) and their amplitude spectra for the chatter regime (example 3). 
preceding section, chatter-free power spectra are relatively flat, whereas chatter power spectra exhibit a strong peak at the chatter frequency. According to the properties of the entropy (Eq. (1)), it is expected that $H^{\prime}(S)$ should be lower for chatter than that for chatter-free regime. The results shown in Fig. 8 agree with these expectations. For the chatter-free example (example 1), entropy of the normal force spectra $H^{\prime}\left(S_{F_{\mathrm{n}}}\right)$ is approximately constant throughout all the eight plunges. In example 2, the decrease of $H^{\prime}\left(S_{F_{\mathrm{n}}}\right)$ in plunges 2-5 correlates well with the rise of chatter vibrations (Fig. 5). Similarly in example 3 , low values of $H^{\prime}\left(S_{F_{\mathrm{n}}}\right)$ in plunges 2, 5, 6 correctly indicate chatter. Values of the entropy of the RMS acoustic emission spectra $H^{\prime}\left(S_{\mathrm{AE}_{\mathrm{RMS}}}\right)$ are generally higher than values of $H^{\prime}\left(S_{F_{\mathrm{n}}}\right)$. This could be expected because the amplitude of the chatter peak in $\mathrm{AE}_{\mathrm{RMS}}$ spectra is not as large compared to that of the other peaks as it is in $F_{\mathrm{n}}$ spectra. Nevertheless, in chatter examples, $H^{\prime}\left(S_{\mathrm{AE}_{\mathrm{RMS}}}\right)$ has distinctly lower values in plunges 6-8 (example 2), and plunge 2 (example 3), which correspond to the cases of strongest chatter vibrations. The dashed horizontal lines in Fig. 8 denote the suggested threshold values of $H^{\prime}$ which could be used for the automatic detection of chatter. The thresholds were set based on spectra from 50 workpieces in order to ensure early detection of chatter and avoid false alarms. A comparison of $H^{\prime}$ for $F_{\mathrm{n}}$ and $\mathrm{AE}_{\mathrm{RMS}}$ with respect to the corresponding thresholds confirms that the normal force is more sensitive to chatter vibration than RMS acoustic emission.

The second chatter indicator proposed in Section 2 is
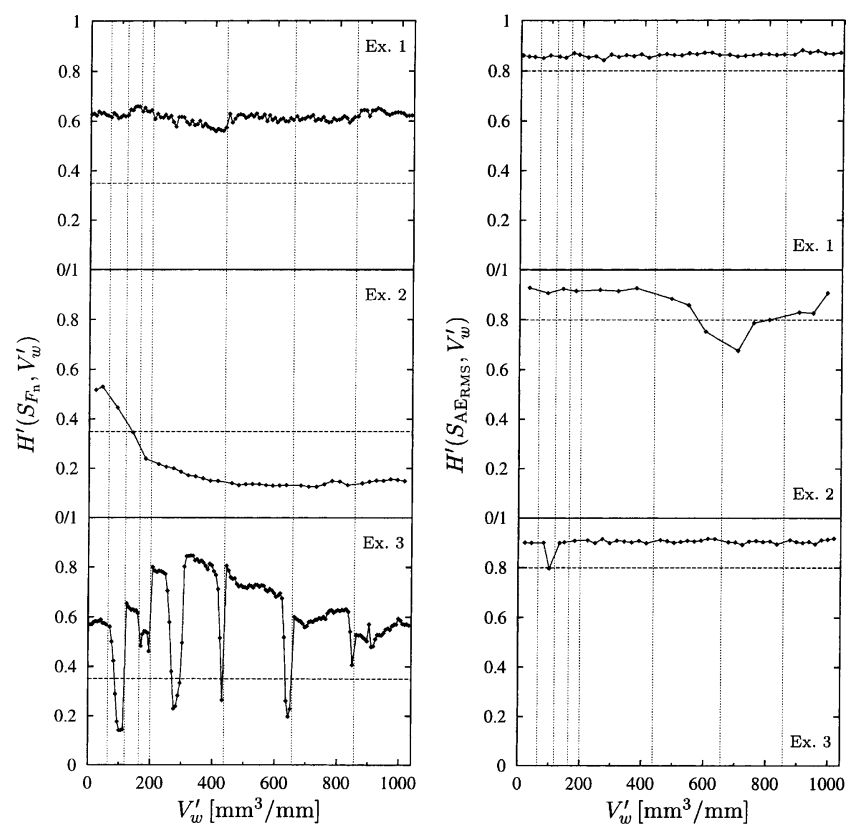

Fig. 8. Normalized entropy of power spectra of the normal grinding force (left) and the RMS acoustic emission. The vertical dotted lines separate the plunges. The horizontal dashed lines denote the threshold values, $H^{\prime}\left(S_{F_{\mathrm{n}}}\right)=0.35$ (left) and $H^{\prime}\left(S_{\mathrm{AE}_{\mathrm{RMS}}}\right)=0.8$. the normalized coarse-grained information rate (NCIR). Here, NCIR is calculated from the fluctuations of the normal grinding force and RMS acoustic emission. For both $F_{\mathrm{n}}$ and $\mathrm{AE}_{\mathrm{RMS}}$, random-like fluctuations are typical of chatter-free regime (resulting in a flat power spectrum), while nearly harmonic fluctuations are typical of chatter regime (spectrum with a strong peak). As a measure of predictability, NCIR is therefore expected to assume lower value in chatter-free than in chatter regime. Chatter detection results using NCIR are shown in Fig. 9. In example 1, NCIR of the normal force, $\operatorname{NCIR}\left(F_{\mathrm{n}}\right)$, has values close to 0 in all the eight plunges, as it should be in a chatter-free regime. In example 2, $\operatorname{NCIR}\left(F_{\mathrm{n}}\right)$ increases in plunges $2-5$, correctly indicating the slow rise of chatter, whereas the "jumps" of $\operatorname{NCIR}\left(F_{\mathrm{n}}\right)$ in example 3 match well with short bursts of chatter. NCIR for fluctuations of $\mathrm{AE}_{\mathrm{RMS}}$ has significantly lower values than for $F_{\mathrm{n}}$. Similar to the entropy of $\mathrm{AE}_{\mathrm{RMS}}$ spectra, NCIR ( $\left.\mathrm{AE}_{\mathrm{RMS}}\right)$ increases only in plunges with strong chatter vibrations. For the purpose of automatic chatter detection, threshold values for NCIR can be set. The threshold in Fig. 9 was set such that the discriminating powers of both NCIR and $H^{\prime}$ (in Fig. 8) against chatter are approximately the same.

\section{Discussion and conclusions}

The article proposes the application of entropy and CIR for automatic chatter detection in grinding. Entropy
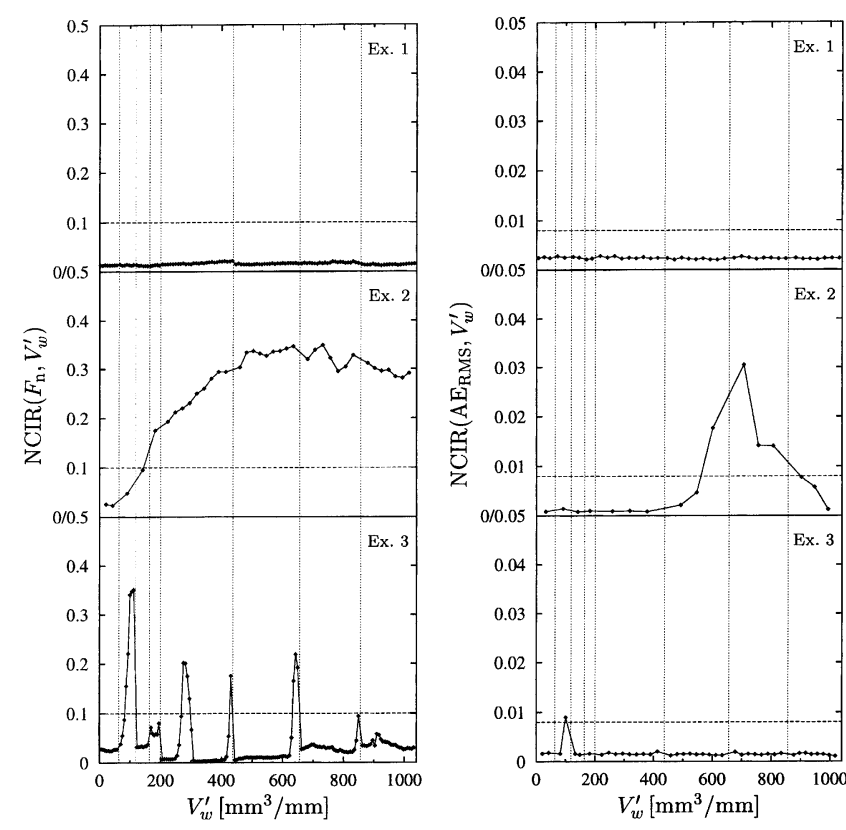

Fig. 9. Normalized coarse-grained information rate for the normal grinding force (left) and the RMS acoustic emission. Note the difference in vertical scales of left and right graphs. The vertical dotted lines separate the plunges. The horizontal dashed lines denote the threshold values, $\operatorname{NCIR}\left(F_{\mathrm{n}}\right)=0.1$ (left) and $\operatorname{NCIR}\left(\mathrm{AE}_{\mathrm{RMS}}\right)=0.008$. 
and CIR are both defined in the information theory. For the purpose of chatter detection, entropy is calculated from the power spectra, while CIR is calculated from the fluctuations of the measured quantity. Normalization of entropy and CIR limits their values to the interval $[0,1]$, which enables setting of a threshold needed for automatic chatter detection. Entropy and CIR are verified as chatter indicators in outer diameter plunge feed grinding, using signals of the normal grinding force and RMS acoustic emission. The results show that entropy and CIR perform equally well as chatter indicators. When calculated from the normal force signals, they indicate the chatter already in its early, developing stage, even before the workpiece has been damaged. In contrast, when calculated from the RMS acoustic emission signals, they indicate only the cases of strong, fully developed chatter, which cause pronounced waviness of the workpiece roundness. Based on the experimental tests, threshold values of entropy and CIR are suggested which reliably discriminate between chatter-free and chatter grinding regimes.

There are several properties of the indicators proposed that render them appropriate for on-line automatic chatter detection. First of all, only scalar values have to be monitored. This is more convenient for automation than the monitoring of multicomponent power spectrum as in the case of spectral analysis. Second, chatter is detected regardless of the chatter frequency. However, it is reasonable to make a test run with chatter in order to estimate approximately the order of magnitude of the chatter frequency so that an appropriate sampling rate for the measured signals can be selected. Alternatively, the range of possible chatter frequencies can also be inferred from the phase of the machine-tool's transfer function [12]. Third, because of the normalization of the indicators, a threshold for automatic detection can be set. The value of the threshold depends on the signals used to calculate the indicators and on the desired sensitivity of the monitoring system. Finally, the calculation of the entropy and CIR is computationally simple. The time required to acquire the signals and calculate the indicators depends on the length of the signals. In this article, all the calculations were performed on signals comprising 32,768 (normal force, sampled at $20 \mathrm{kHz}$ ) and 10,485 points ( $\mathrm{AE}_{\mathrm{RMS}}$, sampled at $10 \mathrm{kHz}$ ). Extensive numerical experiments have revealed that satisfying results can be achieved with the normal force signals containing as few as 2000 points [6]. Processing of such short signals can be carried out in a few tenths of a second which is fast enough for on-line monitoring.

The major cause of false alarms in the proposed chatter detection methods is the harmonic vibrations in the measured signals which become pronounced in the absence of chatter vibrations but are not associated with them. A possible source of such vibrations is the unbalance of the wheel and/or workpiece which is reflected in power spectra as peaks at the wheel and workpiece revolution frequencies and a few of their higher harmonics. Since these frequencies are usually known in advance, they can be suppressed by a high-pass filter.

Finally, note that the onsets of chatter vibrations in grinding and turning most probably represent a dynamically analogous phenomenon which is described in dynamics theory as a Hopf bifurcation [13]. Consequently, the methods for chatter detection presented in this article are expected to be useful for turning as well [14].

\section{Acknowledgements}

The authors acknowledge the support of the Volkswagen Foundation from Germany and EU COST Action P4. JG, EG and IG also acknowledge the support of the Slovene Ministry of Education and Science.

\section{References}

[1] I. Inasaki, B. Karpuschewski, H.-S. Lee, Grinding chatter-origin and suppression, Annals of the CIRP 50 (2) (2001) 515-534.

[2] H.K. Tönshoff, T. Friemuth, J.C. Becker, Process monitoring in grinding, Annals of the CIRP 51 (2) (2002) 1-21.

[3] D.-W. Cho, S.J. Lee, C.N. Chu, The state of machining process monitoring research in Korea, International Journal of Machine Tools and Manufacture 30 (11) (1999) 1697-1715.

[4] B. Karpuschewski, M. Wehmeier, I. Inasaki, Grinding monitoring system based on power and acoustic emission sensors, Annals of the CIRP 49 (1) (2000) 235-240.

[5] E. Govekar, A. Baus, J. Gradišek, F. Klocke, I. Grabec, A new method for chatter detection in grinding, Annals of the CIRP 51 (1) (2002) 267-270.

[6] A. Baus, Potentiale der Nichtlinearen Zeitreihenanalyse bei der Anwendung auf Schleifprozeßsignale, PhD Thesis, RWTH Aachen, Germany (2002).

[7] T.M. Cover, J.A. Thomas, Elements of Information Theory, Wiley Series in Telecommunications, John Wiley \& Sons, Inc., New York, 1991.

[8] E. Ott, Chaos in Dynamical Systems, Cambridge University Press, 1993

[9] H. Kantz, T. Schreiber, Nonlinear Time Series Analysis, Cambridge Nonlinear Science Series, Vol. 7, Cambridge University Press, Cambridge, 1997

[10] M. Paluš, V. Komárek, Z. Hrnčiř, K. Štěrbová, Synchronization as adjustment of information rates: Detection from bivariate time series, Physical Review E63 (04-6211).

[11] M. Paluš, Coarse-grained entropy rates for characterization of complex time series, Physical D 93 (1-2) (1996) 64-77.

[12] M. Weck, Werkzeugmaschinen-Fertigungssysteme Band 4: Meßtechnische Untersuchung und Beurteilung, VDI Verlag, 1996.

[13] S.H. Strogatz, Nonlinear Dynamics and Chaos, Addison-Wesley, Reading, 1994.

[14] J. Gradišek, E. Govekar, I. Grabec, Using coarse-grained entropy rate to detect chatter in cutting, Journal of Sound and Vibration 214 (5) (1998) 941-952. 